\title{
A Unifying Model for the Substitutional Genetic Load
}

\author{
F. M. Phelps IV \\ 290 Cedar Dr., Mt. Pleasant, Michigan 48848, USA
}

[Received 16 January 1990 and in revised form 10 October 1990]

\begin{abstract}
In this paper, a general deterministic model for gene substitution at all segregating loci is derived. The results of a rigorous mathematical analysis of the equations are stated and found to be consistent with results derived by making typical simplifying assumptions. All previous deterministic calculations of possible rates of gene substitution and the 'substitutional genetic load' are shown to be special cases of the model. New expressions for the substitutional genetic load and rate of substitution in hard selection with $c$ th-order epistasis are given. In addition, the concept of 'threshold selection' is generalized to less extreme 'rank selection', and formulae for the relationship between the substitution rate and the selection coefficient are given. The 'load argument' for the neutral theory is reviewed in light of the new findings and strongly rejected. Several other areas of disagreement, such as the effect of population dynamics and competition on the substitutional genetic load, are sorted out using the model. Some remarks regarding experimental input are made.
\end{abstract}

Keywords: cost of natural selection; epistasis; genetic loads; hard, soft, and rank selection; neutral theory; rate of evolution.

\section{Introduction}

BEFORE the advent of protein and DNA sequencing techniques, Haldane (1957) attempted to compute a theoretical upper limit to the rate of evolution based on what he called the 'cost of natural selection'. His observation was that, in order for allele $B$ to replace allele $A$ in a population, all carriers of $A$ must be weeded out. Now if rapid evolution is proceeding at many loci simultaneously, there is a limit to how many individuals can be wiped out each generation if the population is to survive. Haldane computed how many such 'selective deaths' were required during the process of a single gene substitution, and found, remarkably, that the answer was quite large and essentially independent of the selection coefficient. He argued that such 'selective deaths' place a limit on the rate at which inferior alleles can be weeded out and therefore a limit on the rate of gene substitution. He then made what amounted to an assumption of multiplicative fitnesses and concluded that evolution could not proceed at a rate much more than 1 substitution every 300 generations.

His argument, which led many astray (and continues to do so (Hoyle \& Wickramasinghe, 1986: pp. 21-2)), received renewed attention when Kimura (1968) used the discrepancy between the rate of evolution estimated from protein sequence data ( 2 substitutions per generation) and Haldane's calculation (less than 1 substitution per 300 generations) to propose the neutral theory. See Kimura (1983) for a historical summary. During the period from 1968 to 1975 , 
many papers were written, some attacking and some defending Haldane's calculation. Although those disagreeing with Haldane clearly had a stronger case, there was, and certainly remains, much confusion as to the proper resolution of the issue. Perhaps the most important contribution of the present paper is the general model derived in Section 2 which encompasses all previous deterministic calculations of the substitutional genetic load as special cases. In Section 3, approximate formulae for the selection coefficient and average population fitness are derived. In Section 4, the crucial concepts of 'hard', 'soft', and 'rank' selection are given precise definitions. In Section 5, the results of the analysis of the model equations are stated. They are shown to be consistent with the approximations made in Section 3. In Section 6, previous work is considered and each model is found to be a special case of the general equations derived here. In Section 7, several new results are discussed. In Section 8 , several areas of confusion in the load literature are cleared up. Finally, in Section 9, the question of what actually happens in nature is addressed and some comments regarding experimental input are made.

\section{The model equations}

At issue is the substitution rate $r$ measured in DNA base changes (substitutions, insertions, and deletions) per generation. In the model used below, and also by other authors, favoured mutants will attain fixation (frequency equal to one) only after infinite time. Thus $r$ must be defined as the rate at which favoured alleles cross some arbitrary proportion (say 0.99 ) in the population.

The process of gene substitution is a stochastic one, but, until we have a clear understanding of the deterministic case, there is no point in considering stochastic complications. Using diffusion equations, Kimura \& Maruyama (1969) found that including stochastic effects increases the 'selective deaths' required, as it counts those wasted on favoured alleles which ultimately go extinct. However, their work applies only to a single locus unless the assumption of multiplicative fitnesses is made.

It should be noted that the motivation for the model derived below was to incorporate all previous work as special cases of one set of very general equations, and to do so in a mathematically precise way. The result is an example of how the precision of careful (not difficult) mathematics can be used to sort out several areas of controversy in a biological problem.

\subsection{Haploids}

The model derived here will apply to sexually reproducing haploids. Modifications for diploidy will be considered in the next subsection.

We seek equations for a steady flux of substitution at rate $r$. Equations for the case where $r$ depends on $t$ are given in Phelps (1989).

Since the model is deterministic, every favourable allele will spread, so that the rate of origination of favourable alleles, $R$ alleles per generation, must equal the substitution rate $r$. We assume that each favourable allele arises at a new locus. 
Thus, each generation, substitution begins at $R=r$ new loci. Each substituting locus $L_{j, k}$ can be identified by two parameters, the number of generations $j$ since the superior allele at that locus first appeared and $k=1, \ldots, r$ which specifies to which one of the $r$ such loci we are referring. Note that the first subscript of a locus increases generation by generation. Let the two alleles at $L_{j, k}$ be denoted $\mathscr{S}_{j, k}$ (superior) and $\mathscr{S}_{j, k}$ (inferior). Let $p_{j, k}$ denote the frequency of $\mathscr{S}_{j, k}$. In a steady flux, $p_{j, k}$ is independent of $t$.

We assume that all $\mathscr{S}$ alleles are equally advantageous and that the fitness of an individual is a function of the number of inferior alleles it possesses. This 'identical advantages' assumption does not alter our results, as is discussed below, but it greatly simplifies the details of the theory. We also assume that the initial frequency $p_{0, k}$ is a certain constant $p_{0}$ for all $k$. This being the case, $p_{j, k}$ is independent of $k$ for all $j$ and will be denoted $p_{j}$ where appropriate.

We assume random mating and no linkage. The no-linkage assumption is not as bad as it may appear because the probability that any two segregating loci chosen at random are linked is very small. It seems unlikely that the loci which are linked will introduce enough of a systematic bias to alter the conclusions of this paper, but that must be left as an open question.

Let $Z$ be the number of inferior alleles an offspring receives from its parents. Let $B_{j, k}$ be one if $L_{j, k}$ is inferior and zero otherwise, so that

$$
Z=\sum_{j=0}^{\infty} \sum_{k=1}^{r} B_{j, k} .
$$

Assuming Mendelian segregation, the allele at $L_{j, k}$ in an offspring should be $\mathscr{S}_{j, k}$ with probability $p_{j}$ so that $B_{j, k}$ is one with probability $q_{j}=1-p_{j}$. Thus $Z$ is a random variable and an individual's fitness depends on the integer $n$ that $Z$ turns out to be. The assumption that there is no linkage means that the $B_{j, k}$ 's are independent. In order to keep the problem deterministic, we assume that the fraction of individuals that actually receive $n$ inferior alleles is exactly $\mathrm{P}[Z=n]$, the probability that $Z$ is $n$.

We assume that selection operates through differential viability. 'Fitness' means the fraction of individuals in the class specified that survive to breed. Let $w(n)$ be the fitness as a function of $n$, the number of inferior alleles an individual has. Because fitness is a viability, $0 \leqslant w(n) \leqslant 1$. The average population viability $V$ is given by

$$
V=\sum_{n=0}^{\infty} w(n) \mathrm{P}[Z=n]
$$

In order to derive the equation for gene frequency change, it is necessary to compute the advantage due to having a superior allele at a specified locus. Let $V_{j}^{+}$denote the expected viability of an individual conditional upon having the superior allele at $L_{j, k}$. (Note that $V_{j}^{+}$is independent of $k$.) Then

$$
V_{j}^{+}=\sum_{n=0}^{\infty} w(n) \mathrm{P}\left[Z=n \mid B_{j, k}=0\right],
$$

where the new notation denotes conditional probability. Let $\mathrm{P}[Z=n]$ and 
$\mathrm{P}\left[Z-B_{j, 1}=n\right]$ be abbreviated by $P(n)$ and $Q_{j}(n)$, respectively. Now

$$
\mathrm{P}\left[Z=n \mid B_{j, k}=0\right]=\mathrm{P}\left[Z-B_{j, k}=n \mid B_{j, k}=0\right]=Q_{j}(n)
$$

by independence. By conditioning on $n$,

so that

$$
P(n)=p_{j} Q_{j}(n)+q_{j} Q_{j}(n-1)
$$

and

$$
Q_{j}(n)-P(n)=q_{j}\left[Q_{j}(n)-Q_{j}(n-1)\right]
$$

$$
V_{j}^{+}-V=q_{j} \sum_{n=0}^{\infty} w(n)\left[Q_{j}(n)-Q_{j}(n-1)\right]
$$

This equation shows the increase in viability conferred upon an individual by the favourable allele at $L_{j, k}$.

Next, we consider the population dynamics. Let $N(t)$ be the population size in generation $t$ and let $B$ be the per capita birth rate of the population. The population equation is

$$
N(t+1)=N(t) B V
$$

The equation relating $p_{j}$ and $p_{j+1}$ is

$$
N(t) B p_{j} V_{j}^{+}=N(t+1) p_{j+1} .
$$

To see this, note that the left-hand side is the number of offspring with allele $\mathscr{S}_{j, k}$ in generation $t$ times their viability, whereas the right-hand side is the number of those alleles (now labelled $\mathscr{S}_{j+1, k}$ ) in the $(t+1)$ th generation. Using (2) and (3), we arrive at

$$
V_{j}^{+} p_{j}=V p_{j+1} .
$$

Letting $\Delta p=p_{j+1}-p_{j}$ and substituting into (1), we obtain

$$
\Delta p=p_{j} q_{j}\left\{\frac{\sum_{n=0}^{\infty} w(n)\left[Q_{j}(n)-Q_{j}(n-1)\right]}{V}\right\}
$$

Let the factor in braces be denoted $s_{j}$. This simplifies the above to the standard equation for gene frequency change,

$$
\Delta p=s_{j} p_{j} q_{j}
$$

Here, $s_{j}$ is called the 'selection coefficient'. The system of equations is now fully specified. In summary, the important equations are equation (4) above,

$$
V=\sum_{n=0}^{\infty} w(n) P(n),
$$

and

$$
s_{j} V=\sum_{n=0}^{\infty} w(n)\left[Q_{j}(n)-Q_{j}(n-1)\right]
$$


where $\quad Z=\sum_{j=0}^{\infty} \sum_{k=1}^{r} B_{j, k}, \quad P(n)=\mathrm{P}[Z=n], \quad$ and $\quad Q_{j}(n)=\mathrm{P}\left[Z-B_{j, 1}=n\right]$. Equations (5) and (6) will be called the 'viability' and 'selection' equations, respectively.

\subsection{Diploids}

The derivation of the viability and selection equations in the diploid case parallels the haploid case, but differs in several details. In this section, the derivation is outlined.

Let the segregating loci be labelled as before. In a diploid species, the three genotypes at $L_{j, k}$ are $\mathscr{S}_{j, k} \mathscr{\Im}_{j, k}, \mathscr{S}_{j, k} \mathscr{S}_{j, k}$, and $\mathscr{S}_{j, k} \mathscr{S}_{j, k}$. Let these three genotypes be labelled B (bad), H (heterozygote), and G (good), respectively.

In the diploid case, we assume that the fitness of an individual is a function of the vector $A=(X, Y)$, where $X$ is the number of type B loci, and $Y$ is the number of type $\mathrm{H}$ loci the individual has. Thus we let $w(m, n)$ denote the fitness of an individual for which $\boldsymbol{A}$ is $(m, n)$.

To determine $\boldsymbol{A}$ for an offspring, let the random vectors $\boldsymbol{C}_{j, k}$ be independent with distribution

$$
C_{j, k}= \begin{cases}(0,0) & \text { with probability } p_{j}^{2} \\ (0,1) & \text { with probability } 2 p_{j} q_{j}, \\ (1,0) & \text { with probability } q_{j}^{2}\end{cases}
$$

Here it is assumed that $p_{j, k}$ is independent of $k$ as in the haploid case. Then

$$
\boldsymbol{A}=(X, Y)=\sum_{j=0}^{\infty} \sum_{k=1}^{r} C_{j, k}
$$

It is important to note that $X$ is not independent of $Y$.

In order to derive a selection equation, it is necessary to compare the conditional viability of an individual given a certain genotype at locus $L_{j, k}$ with the average population viability. Let $V_{j}^{d}$ be the expected viability of an individual given that $L_{j, k}$ is type $d$, where $d$ is one of the three genotypes $\mathrm{B}, \mathrm{H}$, or $\mathrm{G}$. Let $\mathrm{P}[\boldsymbol{A}=(m, n)]$ and $\mathrm{P}\left[\boldsymbol{A}-C_{j, 1}=(m, n)\right]$ be denoted by $P(m, n)$ and $Q_{j}(m, n)$, respectively. Following the method used to compute $V_{j}^{+}$in the previous section, it can be shown that

$$
V_{j}^{\mathrm{O}}=\sum_{n=0}^{\infty} \sum_{m=0}^{\infty} w(m, n) Q_{l}(m, n)
$$

and that

$$
V_{j}^{\mathrm{H}}=\sum_{n=0}^{\infty} \sum_{m=0}^{\infty} w(m, n) Q_{j}(m, n-1)
$$

By definition, the viability equation is

$$
V=\sum_{n=0}^{\infty} \sum_{m=0}^{\infty} w(m, n) P(m, n) .
$$


The population equation (2) can be used to show that

$$
\Delta p_{j}=\frac{p_{j}}{V}\left(p_{j} V_{j}^{\mathrm{G}}+q_{j} V_{j}^{\mathrm{H}}-V\right)
$$

where $\Delta p_{j}=p_{j+1}-p_{j}$. Using the above formulae, equation (7) can be written as

$$
\Delta p_{j}=u_{j} p_{f} q_{j}
$$

where $u_{j}$ is given by

$$
u_{j}=\frac{1}{V} \sum_{n=0}^{\infty} \sum_{m=0}^{\infty} w(m, n)\left[\left(2 p_{j}-1\right) \Delta_{y} Q_{j}(m, n)+q_{j} \Delta_{x} Q_{j}(m, n)\right]
$$

and $\Delta_{x} Q_{j}(m, n)$ is defined by

$$
\Delta_{x} Q_{j}(m, n)=Q_{j}(m, n)-Q_{j}(m-1, n),
$$

while $\Delta_{y} Q_{j}(m, n)$ is defined by

$$
\Delta_{y} Q_{j}(m, n)=Q_{j}(m, n)-Q_{j}(m, n-1) .
$$

Equation (8) is the diploid version of the selection equation.

\section{The integral approximations}

In this section, we make an approximation suggested by the central limit theorem (CLT), and obtain simple expressions which approximate the integral and viability equations. For simplicity, we will analyse the haploid equations and merely state the corresponding results for the diploid case.

\subsection{Haploids}

The key to analysing the equations is to note that $Z$ (or even $Z-B_{j, k}$ ) is a sum of independent (but not identically distributed) random variables and therefore (as suggested by the CLT) is distributed approximately normally. Exact conditions under which this is a good approximation are given in Section 5. We only remark here that the CLT provides a good approximation if the typical selection coefficient is small relative to the substitution rate.

Let $f(x)$ be the density function for an $\mathrm{N}(0,1)$ random variable, that is

$$
f(x)=\frac{1}{(2 \pi)^{\frac{1}{2}}} \mathrm{e}^{-x^{2} / 2}
$$

If there is a simple formula for $w(n)$ which can be interpreted as a function of all $n$, not just the positive integers, the viability equation is approximated by

$$
V \approx \frac{1}{\sigma} \int_{0}^{\infty} w(x) f\left(\frac{x-\mu}{\sigma}\right) \mathrm{d} x,
$$

where $\mu$ and $\sigma^{2}$ are the mean and variance of $Z$. 
If the difference is approximated by a derivative, the selection equation becomes

$$
s_{j} \approx \frac{1}{\sigma_{j}^{2} V} \int_{0}^{\infty} w(x) f^{\prime}\left(\frac{x-\mu_{j}}{\sigma_{j}}\right) \mathrm{d} x,
$$

where $\mu_{j}$ and $\sigma_{j}^{2}$ are the mean and the variance of $Z-B_{j, k}$, respectively. However, if $\sigma$ is large (which it must be for the CLT to apply), the selection equation (and so $s_{j}$ ) is virtually independent of $j$ and

$$
s \approx \frac{1}{\sigma^{2} V} \int_{0}^{\infty} w(x) f^{\prime}\left(\frac{x-\mu}{\sigma}\right) \mathrm{d} x .
$$

These approximations are not new: they are given in Kimura \& Crow (1978) in the context of the mutation load. The significance in deriving them from the above model is that, if a rigorous justification of these approximations is needed, the starting point for that argument is specified.

In order to use these equations, we must calculate $\mu$ and $\sigma^{2}$. Since the $B_{j, k}$ 's are independent,

$$
\mu=r \sum_{j=0}^{\infty} q_{j} \text { and } \sigma^{2}=r \sum_{j=0}^{\infty} p_{j} q_{j}
$$

If the selection coefficient $s$ is small and essentially independent of $j$, the difference equation (4) can be replaced by the differential equation

$$
\frac{\mathrm{d} p}{\mathrm{~d} t}=s p q
$$

Then

$$
\sum_{j=0}^{\infty} q_{j} \approx \int_{t=0}^{\infty} q(t) \mathrm{d} t=\frac{1}{s} \int_{p_{0}}^{1} \frac{\mathrm{d} p}{p}=\frac{1}{s} \log \left(1 / p_{0}\right) .
$$

Thus

$$
\mu \approx \frac{r}{s} \log \left(1 / p_{0}\right) .
$$

A similar calculation gives

$$
\sigma^{2} \approx \frac{r}{s}\left(1-p_{0}\right)
$$

\subsection{Diploids}

In the diploid case, the integral approximation to the viability equation is

$$
V \approx \int_{y=0}^{\infty} \int_{x=0}^{\infty} w(x, y) f_{A}(x, y) \mathrm{d} x \mathrm{~d} y,
$$


while the approximation for the selection equation is

$$
u(p) \approx \frac{1}{V} \int_{y=0}^{\infty} \int_{x=0}^{\infty} w(x, y)\left((2 p-1) \frac{\partial}{\partial y} f_{A}(x, y)+q \frac{\partial}{\partial x} f_{A}(x, y)\right) \mathrm{d} x \mathrm{~d} y,
$$

where $f_{A}(x, y)$ is the density of a bivariate normal random variable with mean and covariance matrix equal to that of $\boldsymbol{A}$.

If $h$ is a measure of dominance in diploids, it is natural to assume that fitness is a function of the quantity $2 Z$, where $Z=X+h Y$. The factor of 2 is unnecessary, but natural, so that, in the case of no dominance $\left(h=\frac{1}{2}\right), 2 Z$ is exactly the number of inferior alleles. This means that there is a function $\omega$ such that, for every $(x, y)$ pair, $\omega(2 z)=w(x, y)$, where $z=x+h y$. If this is the case, it is possible to reduce the selection and viability integrals to one dimension. The results are

$$
V \approx \frac{1}{\grave{\sigma}} \int_{0}^{\infty} \omega(2 z) f\left(\frac{z-\mu}{\sigma}\right) \mathrm{d} z
$$

and

$$
u \approx \frac{(2 p-1) h+q}{\sigma^{2} V} \int_{0}^{\infty} \omega(2 z) f^{\prime}\left(\frac{z-\mu}{\sigma}\right) \mathrm{d} z,
$$

where $\mu$ and $\sigma^{2}$ are the mean and variance of $Z$. Note that, from this point on, expressions involving $\omega$ refer to diploid populations while expressions involving $w$ as a function of a single variable refer to haploids.

We must calculate $\mu$ and $\sigma$. Let $C_{j, k}^{(i)}$ be the $i$ th component of $C_{j, k}$ and let $D_{j, k}$ be $C(1)+h C_{j, k}^{(2)}$. Then

$$
Z=\sum_{k=1}^{r} \sum_{j=0}^{\infty} D_{j, k}
$$

Thus

$$
\mu=r \sum_{j=0}^{\infty} \mathrm{E}\left[D_{j, 1}\right]=r \sum_{j=0}^{\infty}\left(2 p_{j} q_{j} h+q_{j}^{2}\right),
$$

where $E$ denotes the expected value or mean. Similarly,

$$
\sigma^{2}=r \sum_{j=0}^{\infty}\left[2 p_{j} q_{j} h^{2}+q_{j}^{2}+q_{j}^{2}\left(2 p_{j} h+q_{j}\right)^{2}\right]
$$

Let

$$
s_{j}=\frac{u_{j}}{2\left[\left(2 p_{j}-1\right) h+q_{j}\right]},
$$

so that, when $h=\frac{1}{2}, s_{j}=u_{j}$. The slow selection approximation to the difference equation is now

$$
\frac{\mathrm{d} p}{\mathrm{~d} t}=2[(2 p-1) h+q] s p q
$$


To a good approximation,

$$
\mu=\frac{r}{2 s} I\left(h, p_{0}\right)
$$

where

$$
I\left(h, p_{0}\right)=\frac{1}{1-h}\left(\log \left(1 / p_{0}\right)-h \log \frac{h}{1-h+p_{0}(2 h-1)}\right)
$$

if $h \neq 0$ or 1 . In the case of total dominance of the inferior allele, $h=1$ and

$$
I=\frac{1-p_{0}}{p_{0}}+\log \frac{1}{p_{0}},
$$

which is very large for small $p_{0}$. At the other extreme, if $h=0$, the result reduces to $I=\log \left(1 / p_{0}\right)$. If $h=\frac{1}{2}$, then $I=2 \log \left(1 / p_{0}\right)$. The important quantity $I$ is plotted as a function of $h$ for $p_{0}=\mathrm{e}^{-10}$ in Fig. 1. Note that it blows up as complete dominance, $h=1$, is approached.

By the same method, the variance is found to be

$$
\sigma^{2}=\frac{r}{2 s} J\left(h, p_{0}\right)
$$

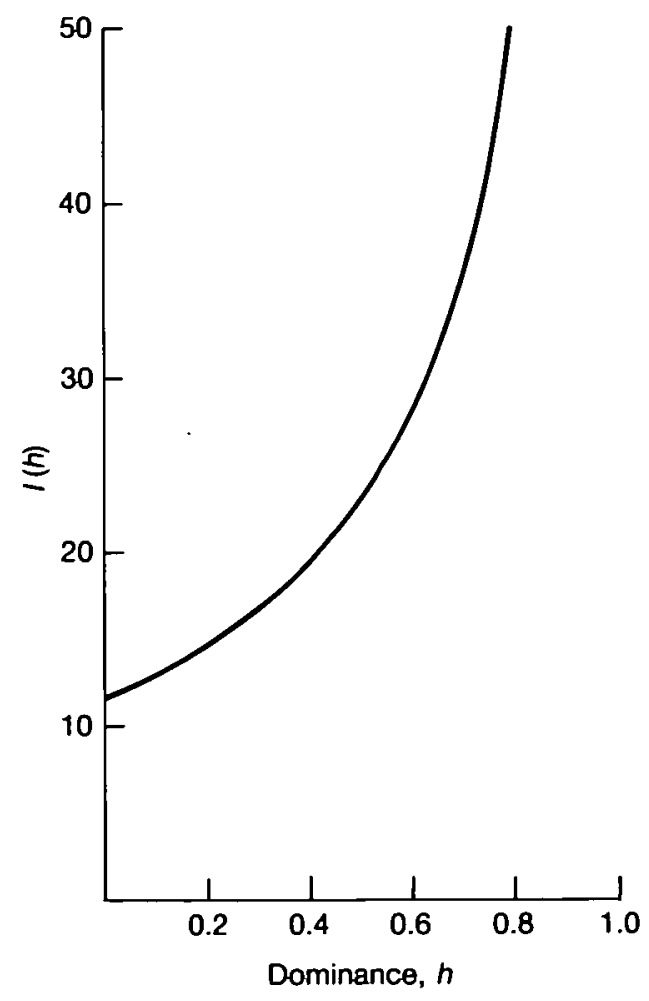

FIG. 1. The function $I(h)$ for $p_{0}=10^{-5}$. 


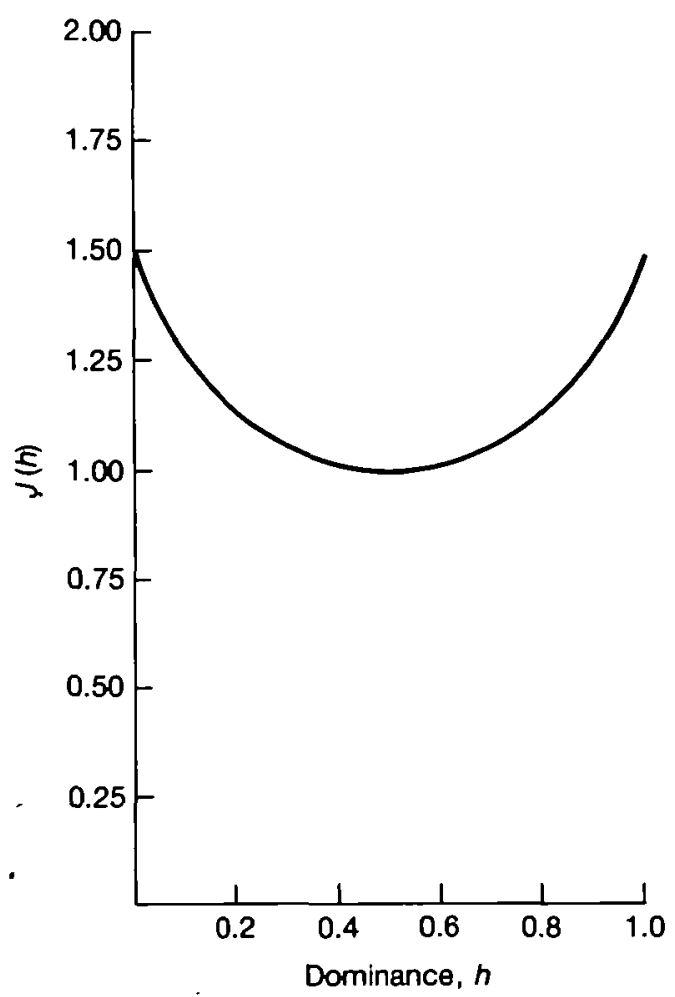

Fic. 2. The function $J(h)$ for $p_{0}=10^{-5}$.

where

$$
J\left(h, p_{0}\right)=\frac{2 h(1-h) \log \left(\frac{1-h+p_{0}(2 h-1)}{h}\right)+(2 h-1)\left(1-p_{0}\right)\left[3+p_{0}(2 h-1)\right]}{2(2 h-1)}
$$

if $h \neq 0$ or $\frac{1}{2}$. Since $p_{0}$ is likely to be extremely small, this is approximately

$$
\frac{h(1-h)}{2 h-1} \log \left(\frac{1-h}{h}\right)+\frac{3}{2}
$$

which is plotted in Fig. 2 . Note that $J$ is not very sensitive to $h$, unlike $I$ above. For $h=\frac{1}{2}$, the formula does not apply, but it is easy to verify that $J\left(\frac{1}{2}, p_{0}\right)=$ $1-p_{0}$. For $h=0$, we find that $J$ is $\frac{1}{2}\left(1-p_{0}\right)\left(3-p_{0}\right)$.

\section{Hard, soft, and rank selection}

The terms 'hard' and 'soft' selection were coined by Wallace (see Wallace, 1975) and have been quite popular in discussions of genetic loads. The term 'rank selection' was used by Wills (1978) in a discussion of the segregational genetic 
load. Since these terms are used in different ways by different authors, it is best to define our usage at the outset. Here, these terms are given precise definitions for the haploid case. The generalization to diploidy is obvious.

The issue is what determines viability. If, in a given environment, the viability of an individual is determined (up to a density-dependent constant factor) by $n$, the number of inferior alleles it carries (without reference to other individuals in the population), then selection is said to be hard.

If selection is hard, fitness has the form

$$
w(n)=w(0) e^{-\varepsilon g(n)}
$$

for some positive function $g$ and some constant $\varepsilon$. Of course, $\varepsilon$ could be absorbed into the function $g$, but we retain it for reasons which will become apparent below. Here, $w(0)=w(0, N)$ is allowed to depend on the population size $N$, but we will not write out this dependence. If selection is not hard, it is said to be soft.

Next, we consider the case where the composition of the population determines an individual's fitness. Suppose an individual has $Z=n$ inferior alleles. It is important to have a measure of how the individual stands relative to the rest of the population. The cumulative distribution function $R(n)$ defined by

$$
R(n)=\mathrm{P}[Z \leqslant n]
$$

is such a measure and $R(n)$ will be called the individual's rank. Note that rank 0 is optimal, while the least fit possible individual has rank 1 . If fitness is a function of rank, that is, if there is a function $G$ such that $w(n)=G(R(n))$, the scheme is said to be rank selection. In such a case, $G$ is called the viability function. Rank selection is a special form of soft selection.

The classical example of hard selection is the law of multiplicative fitnesses, that is, $w(n)=w(0)(1-s)^{n}=w(0) \mathrm{e}^{-m}$, where $\varepsilon=-\log (1-s) \approx s$. The classical example of soft selection is the threshold or truncation rank selection model where the most fit fraction $V$ of the population live and the least fit fraction $1-V$ die. In this case, $G(y)$ is 1 for $y$ less than $V$, and 0 for $V<y \leqslant 1$.

\section{Analysis of the model}

\subsection{Rank selection}

The integral approximation formulae have been established for large $r$ in the case of rank selection with $h=\frac{1}{2}$ in Phelps (1989). The results are stated here for arbitrary $h \in[0,1]$, but the lengthy and technical proof will be omitted.

In order to state the result, we need to define some notation. As before, let $f(x)$ denote the density of an $\mathrm{N}(0,1)$ random variable and let $F(x)$ be its integral, that is,

$$
F(x)=\int_{-\infty}^{x} f(\xi) \mathrm{d} \xi
$$


Let $K$ be the constant defined by

$$
K=\frac{\int_{-\infty}^{\infty} G(F(x)) f^{\prime}(x) \mathrm{d} x}{\int_{-\infty}^{\infty} G(F(x)) f(x) \mathrm{d} x}
$$

Let $s_{+}(r)=\sup _{j} s_{j}(r)$ and $s_{-}(r)=\inf _{j} s_{j}(r)$.

THEOREM 1 (Asymptotic Formulae for Rank Selection) Under any diploid rank selection law with $G(y)$ nonincreasing, nonconstant, and continuous almost everywhere,

1. $\lim _{r \rightarrow \infty} s_{-}(r) / s_{+}(r)=1$,

2. $\lim _{r \rightarrow \infty} r s_{+}(r)=K^{2} / 2 J\left(h, p_{0}\right)$,

3. $\lim _{r \rightarrow \infty} \sigma(r)^{2} / r^{2}=\left[J\left(h, p_{0}\right) / K\right]^{2}$,

4. $\lim _{r \rightarrow \infty} \mu(r) / r^{2}=I\left(h, p_{0}\right) J\left(h, p_{0}\right) / K^{2}$.

The first part shows that $s_{j}$ is essentially independent of $j$. The second part says that $r$ is proportional to $1 / s$, so arbitrarily rapid substitution is possible if $s$ is made sufficiently small. The third and fourth parts give asymptotic expressions for the mean and variance of the number of inferior alleles.

These results can be derived from the integral approximations with

$$
\omega(2 z)=G\left(F\left(\frac{z-\mu}{\sigma}\right)\right)
$$

The same formulae apply in the haploid case with $J=1-p_{0}, I=\log \left(1 / p_{0}\right)$, and the factor 2 in the denominator of the second formula omitted. Applications of these results will be discussed in the following sections.

\subsection{Hard Selection}

For hard selection, the following conjecture has not yet been proved. The exact conditions required on $g$ are still uncertain, but the formulae should hold if $g$ is a polynomial with positive coefficients.

CONJECTURE 1 (Asymptotic Formulae for Hard Selection) Let $g(x)$ be positive and increasing on $[0, \infty)$, with $\lim _{x \rightarrow \infty} g(x) / a x^{c}=1$ and $\lim _{x \rightarrow \infty} g^{\prime}(x) / a c x^{c-1}=1$ for some positive constants $a$ and $c$. Let the fitness function $\omega(x)$ be given by $\omega(x)=\omega(0) \mathrm{e}^{-\varepsilon g(x)}$. Then

1. $\lim _{e \rightarrow 0} s_{-} / s_{+}=1$,

2. $\lim _{\rightarrow \rightarrow 0} s_{+} / \operatorname{eg}^{\prime}(2 \mu)=1$,

3. $\lim _{\rightarrow \rightarrow 0} V / \omega(0) \mathrm{e}^{-g(2 \mu)}=1$,

4. $\lim _{\mathrm{s} \rightarrow 0} \mu s_{+}=\frac{1}{2} r I\left(h, p_{0}\right)$,

5. $\lim _{e \rightarrow 0} \sigma^{2} s_{+}=\frac{1}{2} r J\left(h, p_{0}\right)$.

Again, the first part shows that $s_{j}$ is independent of $j$. The second part gives an asymptotic expression for the selection coefficient which is very different from the 
rank selection case. The third part gives an asymptotic expression for the average population viability. The fourth and fifth parts give the mean and variance.

These results are suggested by the integral approximations (9) and (10) as follows. Let $v=z / \mu$. The viability integral becomes

$$
V=\frac{\mu}{\sigma} \int_{0}^{\infty} \omega(2 \mu v) f\left(\frac{\mu}{\sigma}(v-1)\right) \mathrm{d} v .
$$

In the small- $\varepsilon$ limit, fitness differences are small and so selection is weak. Thus, $\mu / \sigma$ is large (see (11) and (12) above), and so the function

$$
\frac{\mu}{\sigma} f\left(\frac{\mu}{\sigma}(v-1)\right)
$$

acts like the delta-function $\delta(v-1)$. This suggests that, in the limit,

$$
V=\omega(2 \mu)=\omega(0) \mathrm{e}^{-a_{g}(2 \mu)}
$$

which is part 3 in the conjecture above. An integration by parts and the same change of variables yields

$$
s=\frac{\varepsilon}{V} \int_{0}^{\infty} g^{\prime}(2 \mu v) \omega(2 \mu v) f\left(\frac{\mu}{\sigma}(v-1)\right) \mathrm{d} v .
$$

The same delta-function argument and simplification gives

$$
s=\varepsilon g^{\prime}(2 \mu),
$$

which is part 2 above. The formulae for the mean and the variance are just equations (11) and (12) above.

The results for the haploid case are obtained by putting $J=1-p_{0}$ and $I=\log \left(1 / p_{0}\right)$ in the above, dropping the factor 2 in parts $2-5$ in the conjecture, and changing $\omega$ to $w$.

From parts 2 and 4 above, we conclude that $\lim _{e \rightarrow 0} \mu=\infty$. Then parts 2,3 , and 4 imply

$$
r \approx \frac{L}{I\left(h, p_{0}\right)} \lim _{x \rightarrow \infty} \frac{x g^{\prime}(x)}{g(x)}=\frac{c L}{I\left(h, p_{0}\right)},
$$

where $L=\log [\omega(0) / V)]$. The important quantity $L$ is the $\log$ of the ratio of the fitness of the optimal possible individual to the average fitness of the population. In the literature, this quantity (or sometimes the simple ratio $\omega(0) / V$ ) has been called the 'substitutional genetic load'.

This is truly a remarkable formula in that the rate of substitution is independent of $\varepsilon$ and the selection coefficient.

\section{A unification of previous wort}

Although several different quantitative approaches to the substitutional genetic load have been made in the literature, essentially all of them can be seen to be special cases of either hard or rank selection applied to the integral approximations. 


\subsection{Previous Hard Selection Models}

Although Haldane did not fill in the details, he implicitly assumed multiplicative fitnesses, or $\omega(x)=\omega(0) \mathrm{e}^{-x x}$. Kimura (1971 and many other papers) accepted Haldane's assumptions.

With multiplicative fitnesses, $g(x)=x$, so (14) becomes

$$
r \approx \frac{L}{I\left(h, p_{0}\right)}
$$

If the population is constant, $B V=1$, so that $L=\log [\omega(0) B]$. By putting reasonable estimates on $L$ and $p_{0}$ and making allowance for various values of $h$, Haldane (in essence) used this formula to make his famous estimate that $r=\frac{1}{300}$. It is clear that this low estimate bothered him (see Hoyle \& Wickramasinghe, 1984: pp. 194-5), and the whole problem became known as 'Haldane's dilemma' (Maynard Smith, 1968).

The substitution rate as a function of $h$ is plotted for $\omega(0) / V=10$ and $p_{0}=10^{-5}$ in Fig. 3. Although these parameter values are less conservative than those chosen by Haldane, the substitution rate falls far below $r=2$. As the dominance $h$ of the inferior allele increases, substitution grinds to a halt.

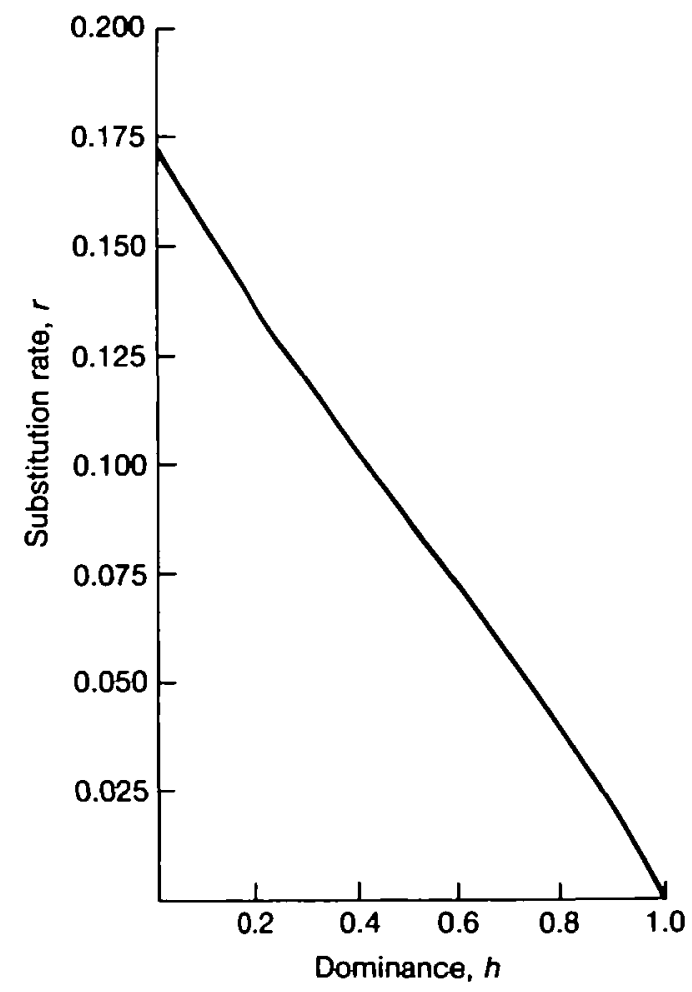

FIG. 3. Rate of substitution as a function of dominance in hard selection with multiplicative fitnesses, $L=\log 10$, and $p_{0}=10^{-5}$. 


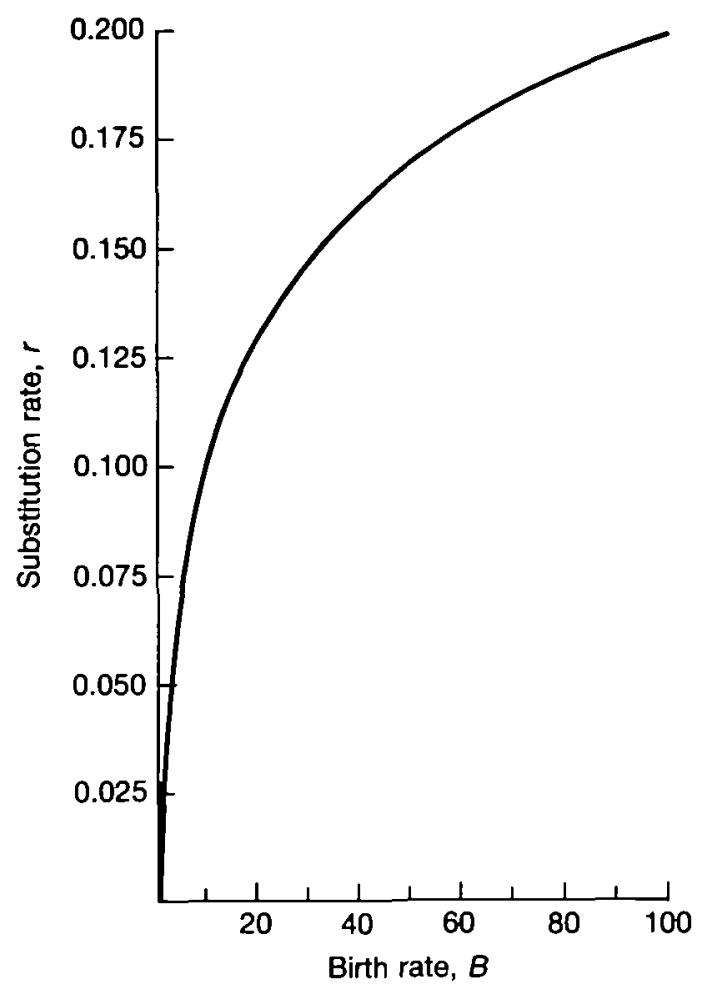

FIo. 4. Rate of substitution as a function of per capita birth rate in hard selection with multiplicative fitnesses, no dominance, and $p_{0}=10^{-5}$.

If $\omega(0)=1$ and the population size is constant, then the substitution rate is a function of the per capita birth rate. This is plotted in Fig. 4 for $p_{0}=10^{-5}$. As can be seen, high fecundity, which allows more selection, results in higher substitution rates, but not as high as $r=2$.

Note that, if there were no inferior alleles, $V$ would be $\omega(0)$. If substitution is going on at a locus, this implies that there is an 'inferior' allele at that locus, and so the fitness $V$ is correspondingly reduced. It may be objected that the favourable mutant is 'superior', while the allele it is replacing is as good as it always was, so that there should not be a decrease in $V$. Yet the only way to increase $V$ is to increase $\omega(0)$, and, by definition, $\omega(0) \leqslant 1$. So, even in the optimal case where $\omega(0)=1$, a substitution rate of $r=2$ implies (for $h=\frac{1}{2}$ and $\left.p_{0}=10^{-4}\right) \log V=-36 \cdot 8$, which is an intolerably low population fitness.

Because Haldane and Kimura both used a hard selection model, it appeared that such a reduction in fitness was a necessary consequence of the weeding out of inferior alleles. Haldane unfortunately called this reduction 'the cost of natural selection', while Kimura introduced the equally misleading terminology 'substitutional genetic load'.

While the terminology suggests that the evolutionary process itself necessitates a reduction in population fitness, it is actually the assumption of hard selection 
which forces the reduction. The failure to come to grips with this subtle distinction is at the root of a lengthy controversy. See Van Valen (1963), Brues (1964), Feller (1967), Brues (1969), Kimura \& Crow (1969), Moran (1970), Nei (1971), Felsenstein (1971), Ewens (1972), Grant \& Flake (1974), and Maynard Smith (1978) for details.

The only author who has attempted a non multiplicative fitnesses hard selection model is O'Donald (1969). In our notation, his fitness function (for haploids) was $w(x)=1-\varepsilon x^{2}$. This choice is, however, impossible as the fitness goes negative for large $x$. O'Donald's function should be replaced by $\mathrm{e}^{-\varepsilon x^{2}}$, which agrees with his function for small $x$. O'Donald's model assumed that the frequency of the superior allele at each segregating locus was a certain constant $p$, and so his work is only of qualitative interest in computing possible rates of gene substitution. He used his model to argue that evolution could be more rapid than Haldane had suggested, while not nearly as rapid as suggested by Maynard Smith's (1968) model, which we consider below.

The correct formula for this case $\left(g(x)=x^{2}\right.$ and $\left.\omega(0)=1\right)$ in diploids is

$$
r \approx \frac{2 L}{I\left(h, p_{0}\right)}
$$

Thus, for fixed $r$, the load is half of what it is under multiplicative fitnesses, or, for fixed $L$, the substitution rate is doubled. This halving of the load agrees with what Kimura \& Maruyama (1966) found in the mutation load.

\subsection{Previous Soft Selection Models}

Sved (1968) was the first to use a quantitative rank selection model to compute possible rates of gene substitution. In our notation, his fitness as a function of rank $R$ is $\omega(2 z)=G(R(z))$, where $G$ is defined by

$$
G(y)= \begin{cases}1 & \text { if } y \leqslant V \\ 0 & \text { if } y>V\end{cases}
$$

If we let $\theta=F^{-1}(V)$, then $K$ defined above in equation (13) becomes $f(\theta) / V$. The rate of substitution is given by

$$
r \approx \frac{1}{s}\left(\frac{f^{2}(\theta)}{2 J\left(h, p_{0}\right) V^{2}}\right) .
$$

This is plotted as a function of $V$ for $s=0.01, h=\frac{1}{2}$, and small $p_{0}$ in Fig. 5. Note that $r=2$ is attained for $V \approx 0.9$, which means about $10 \%$ of the population must die selectively each generation. Thus $r=2$ creates a load $L$ of only $\log (1 / V) \approx$ $0 \cdot 10$, whereas in the multiplicative fitnesses model $L=36 \cdot 8$ !

Sved's work differs in that he assumed only a fraction $\mathrm{H}^{2}$ of the variance would contribute to the selective process. He did this, no doubt, in order to soften the extreme threshold implied by $G$. This fraction is called the heritability, and its effect, for a given $r$, is to reduce the selection coefficient by a factor of $1 / H^{2}$. In order to work heritability into our model, we must change our interpretation of $Z$ 


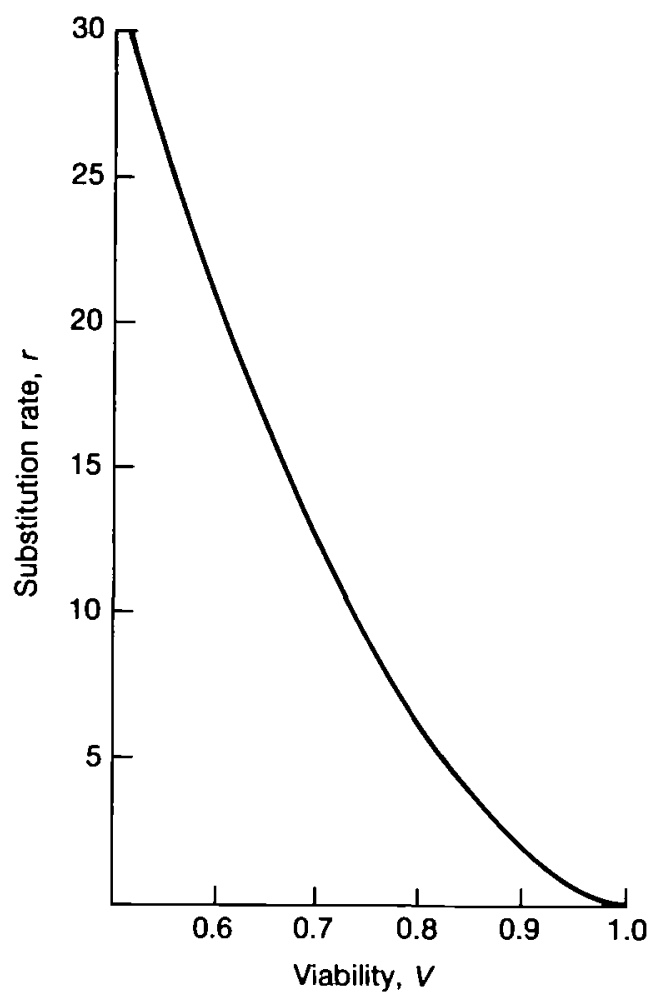

FIG. 5. Rate of substitution as a function of viability in truncation selection in diploids with no dominance and $s=0.01$.

to be some sort of 'fitness potential' that has both a genetic and an environmental component which are independent and additive. The genetic component is assumed to be the quantity $X+h Y$ with variance $\sigma^{2}$ as computed above, while the environmental component is assumed to have mean zero and variance $V_{\mathrm{E}}$ satisfying

$$
H^{2}=\frac{\sigma^{2}}{\sigma^{2}+V_{\mathrm{E}}} .
$$

A more direct way to soften the threshold behaviour is to change $G$, which is done below.

Sved did not plot his version of (15) above, but he did evaluate it for several values of $V, s$, and $H^{2}$. Sved used his numbers to conclude that there is no upper limit to the possible rate of gene substitution. This is now known to be the case in any rank selection model in light of Theorem 1 above, because the substitution rate is inversely proportional to the selection coefficient, which can be arbitrarily small. In contrast, recall that Haldane's bound for $r$ is independent of $s$.

Maynard Smith (1968) used Sved's threshold function $G$ with $V=\frac{1}{2}$ in an influential paper challenging Kimura's load argument for the neutral theory. 
However, he assumed that the superior allele at each segregating locus has frequency $p$ and concluded that, for a wide range of values of $p$, thousands of loci could be selected for simultaneously. With the above formulae, and using Maynard Smith's numbers $\left(s=0.01, p_{0}\right.$ small, and $\left.V=\frac{1}{2}\right)$, we arrive at

$$
r \approx \frac{1}{s}\left(\frac{1}{\pi\left(1-p_{0}\right)}\right) \approx 32
$$

for diploids.

\subsection{Ewen's Work}

Ewens (1972) also considered the substitutional genetic load problem and its implications for possible gene substitution rates in a haploid population. Recall that the definition of the load involves the fitness of the optimal possible individual, $w(0)$. Ewens and others noted that such an optimal individual, that is, an individual with no unfavourable alleles, would never exist in a real population, because $\mathrm{P}[Z=0]$ is vanishingly small for realistic parameter values.

This being the case, he calculated ratio of the fitness of the most fit existing individual to the average population fitness. If $c_{N}$ is the expected value of the largest of $N$ independent $N(0,1)$ random variables, the most fit existing individual in a population of size $N$ will have about $x^{*}=\mu-c_{N} \sigma$ inferior alleles. Although Ewens was sceptical of multiplicative fitnesses, for the sake of argument he assumed they operated over the range of existing individuals. In effect, he chose

$$
w(x)=w\left(x^{*}\right) \mathrm{e}^{-s\left(x-x^{*}\right)}
$$

for all $x$ such that $|x-\mu| \leqslant c_{N} \sigma$. With Kimura's numbers, he computed the ratio $w\left(x^{*}\right) / V$ in a haploid population to be 1.56 , which is not very large at all compared to the ratio $w(0) / V=\mathrm{e}^{36 \cdot 8}$ for the nonexistent optimal individual.

Let us now incorporate this into a load model. First, the above fitness function is an incomplete model. An 'optimal individual' may never arise in nature, but the mere possibility of its existence requires that it be assigned a fitness. For example, such an individual could be created in a future laboratory, and its fitness measured. The natural way to define $w(x)$ for small $x$ in a way which preserves the emphasis of Ewens' calculations is to take $w(x)=w\left(x^{*}\right)$ for $x \leqslant x^{*}$. If this is assumed, $L=\log 1.56$ and rapid evolution is shown to be possible. However, the model is quite artificial, and therefore quite unlikely to describe reality to any useful extent. In particular, it draws attention to the finiteness of the population as opposed to what I suggest is the real issue, that fitness depends strongly on the composition of the population.

Note that this modified Ewen's model is not hard selection because $x-x^{*}$ is not determined by $x$ alone.

\section{New results}

Given the asymptotic formulae in Section 5, several new results are immediately available. 


\subsection{Epistasis in Hard Selection}

Several authors (e.g. Ewens, 1979: p. 254) have pointed out that the genes do not determine fitness independently, as in the multiplicative fitnesses model, but that there is certainly some epistatic interaction which may reduce the load. If $g(x)=x^{c}$ in hard selection, fitness falls off as the $c$ th power of the number of inferior alleles. This case will be called ' $c$ th-order epistasis'. From (14) above, the rate of substitution in a constant population of diploids is

$$
r \approx \frac{c L}{I\left(h, p_{0}\right)},
$$

which is $c$ times the rate Haldane found for multiplicative fitnesses. Equivalently, $L$ is reduced by a factor of $c$. Again, $r$ is independent of the selection coefficient.

Although $r$ is certainly increased over Haldane's estimate, it is impossible to achieve rates of the order of $r=2$ for reasonable parameter values (certainly $c<5$ in nature). It thus appears that this sort of epistasis is unlikely to resolve Haldane's dilemma.

\subsection{Linear Rank Selection}

A serious objection to threshold selection models is that the function $G$ is too extreme. If we relax this function to a linear decay of viability with rank, we have a class of models which will be called 'linear rank selection'. Let

$$
G(y)=-m y+b \text {. }
$$

The constraints are $0<m \leqslant b \leqslant 1$. The average population viability is

$$
V=\frac{1}{2}[G(0)+G(1)]=\frac{1}{2}(2 b-m) .
$$

If we eliminate $b$, we find that $V$ and $m$ may be chosen independently, as long as $0<V<1$ and $m \leqslant 2 V \leqslant 2-m$. The selection integral is computed as follows:

$$
\int_{-\infty}^{\infty} G(F(x)) f^{\prime}(x) \mathrm{d} x=-\int_{-\infty}^{\infty} G^{\prime}(F(x)) f^{2}(x) \mathrm{d} x=\frac{m}{2 \pi} \int_{-\infty}^{\infty} \mathrm{e}^{-x^{2}} \mathrm{~d} x=\frac{m}{2 \sqrt{ } \pi} .
$$

The rate of substitution is given by

$$
r \approx\left(\frac{m^{2}}{8 \pi J\left(h, p_{0}\right)}\right) \frac{1}{s} .
$$

This is plotted as a function of $h$ in Fig. 6 with $s=0.001, m=0.2, V=0.8$, and $p_{0}$ small. Note that the rate does not depend strongly upon $h$, as it does in hard selection, and that substitution is most efficient when there is no dominance $\left(h=\frac{1}{2}\right)$. Also, in order to attain $r=2, s$ needs to be quite small.

For a fixed slope $m$, the substitution rate is maximized (selection is most efficient) when $V$ is minimized at $V=\frac{1}{2} \mathrm{~m}$. In this case, $K^{2}=1 / \pi$. Thus, for linear rank selection, if we think of $s$ as being fixed, the rate of substitution cannot be larger than

$$
r=\left(\frac{1}{2 \pi J\left(h, p_{0}\right)}\right) \frac{1}{s}
$$




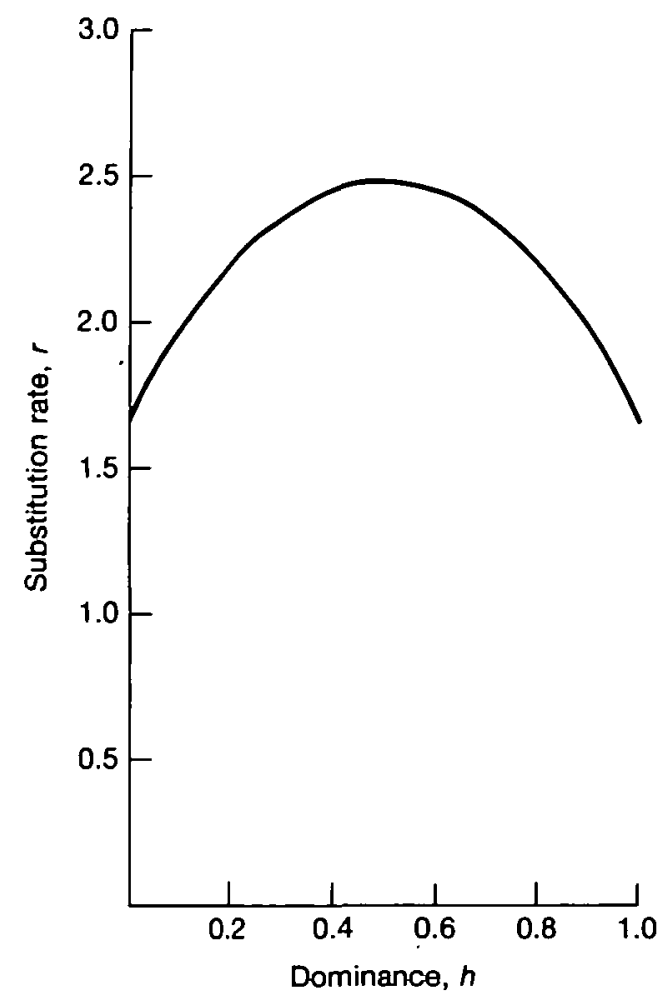

FIG. 6. Rate of substitution as a function of dominance in linear rank selection with $m=0 \cdot 2, V=0 \cdot 8$, and $s=0.001$.

This is in contrast to the case for threshold selection where $K$ blows up as $V \rightarrow 0$. Note also that this maximum for linear rank selection is exactly half the rate in the threshold model with $V=\frac{1}{2}$.

For fixed viability (which is easier to measure than the slope of the supposedly linear viability function), the rate of substitution goes like $m^{2}$. This strong dependence on the slope indicates that selection cannot be too 'gentle' if $r=2$ and $s=0.01$ are to be maintained. The critical value of $m$ in order to attain $r=2$ (for $p_{0}$ small and $h=\frac{1}{2}$ ) is $m=7 \cdot 1 s^{\frac{1}{2}} V$. This is plotted for $s=0.01$ and $s=0.001$ in Fig. 7.

In summary, rapid evolution is possible in linear rank selection, but realistic rates may only be attained either by a steep slope or by taking $s$ to be of the order of 0.001 .

\section{Discussion of controversial issues}

\subsection{Changing Population Size}

A number of people have criticized Haldane's calculation on the basis that it assumes constant population size. It is an easy matter to see how an exponentially 


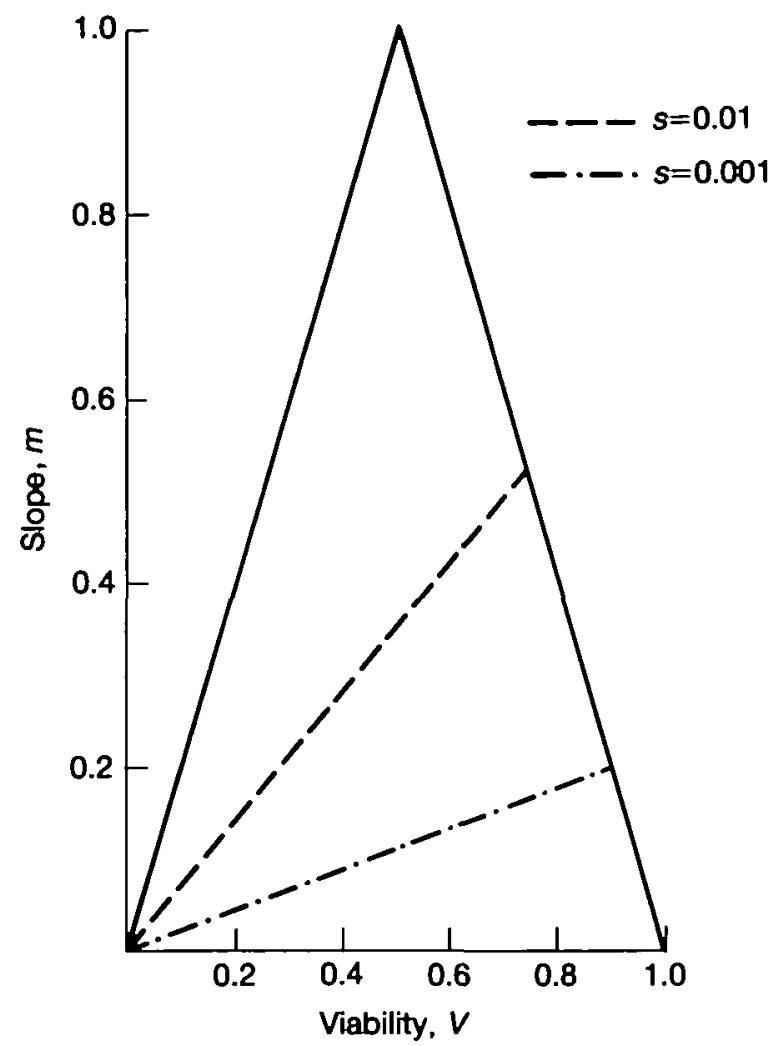

FIo. 7. Slope $m$ necessary to account for $r=2$ as a function of viability in linear rank selection in diploids with no dominance. Allowable parameter values lie below the solid lines.

growing (or decaying) population changes the substitution rate in hard selection. If the population is growing by a factor of $\mathrm{e}^{m}$ each generation, $B V=\mathrm{e}^{m}$, so that the load $L$ is $\log [B \omega(0)]-m$. In the case of $c$ th-order epistasis,

$$
r=\frac{c}{I}\{\log [B \omega(0)]-m\}
$$

The only way to get rapid evolution here is to take $m$ to be large and negative, which implies a population collapse. Despite the arguments of Feller (1967) and Moran (1970), we cannot escape Haldane's dilemma by including population dynamics.

\subsection{The Lag Load}

The most recent paper on the substitutional genetic load is that of Kondrashov (1984). This paper contains a (nonrigorous) proof that the variance of the number of inferior alleles in an individual in a haploid population undergoing threshold selection remains finite for finite $r$. The correct relationship is that $\sigma^{2}$ is proportional to $r^{2}$, which is the fourth part of the rank selection result above. 
Kondrashov also computes what he calls the 'lag load', but he uses such a strange quantity to define it that it is difficult to interpret. The obvious measure of the 'lag load' is $\mu$, the number of inferior alleles per individual when $h=\frac{1}{2}$. The rank selection result above implies that $\mu$ also goes like the substitution rate squared.

\subsection{Is r Really Large?}

It may be objected that the formulae for rank selection only apply in the large $r$ limit, while the debate is whether $r$ is of the order of $\frac{1}{300}$ or 2 . However, the key approximation is the central limit theorem, and it is quite accurate if $\mu$ is significantly larger than $\sigma$. This is the case whenever $r / s$ is of the order of 1 or larger. This is so in all of the above rank selection calculations.

The CLT may not be a good approximation if we really believe $r<\frac{1}{300}$ and $s<0.01$, because in that case so few alleles are undergoing substitution at any given time. However, to arrive at the conclusion that soft selection is compatible with the 'observed' substitution rate, while hard selection is not, we only need to use the CLT for $r=2$.

\subsection{Unequal Effects of the Alleles}

It may be objected that the formulae in this paper depend on the assumption that all inferior alleles have equal effect. Here (for haploids) we outline a demonstration that this is not the case. Suppose fitness is a function of $Z=\sum_{i} a_{i} X_{i}$, where $X_{i}$ is the number of inferior alleles with effect $a_{i}$. Let $s_{i}$ and $r_{i}$ be the selection coefficient and substitution rate, respectively, for alleles of effect $a_{i}$. Then $r=\sum_{i} r_{i}$. The integral approximation for the selection coefficient $s_{i}$ turns out to be $a_{i}$ times what it was before (see Kimura \& Crow, 1978, or derive it by our method above).

Let $\mu_{i}$ and $\mu_{Z}$ be the mean of $X_{i}$ and $Z$, respectively. The result

$$
\mu_{i}=\frac{r_{i}}{s_{i}} \log \left(1 / p_{0}\right)
$$

holds for all $i$ following the method of Section 3.1.

For hard selection,

$$
s_{i}=a_{i} \varepsilon g^{\prime}\left(\mu_{z}\right)
$$

and $L=\varepsilon \mu_{Z}$ as before. Then

$$
\mu_{Z}=\sum_{i} a_{i} \mu_{i}=\frac{g\left(\mu_{Z}\right) \log \left(1 / p_{0}\right)}{g^{\prime}\left(\mu_{Z}\right) L} \sum r_{i},
$$

which implies

$$
r=\frac{L}{\log \left(1 / p_{0}\right)} \lim _{x \rightarrow \infty} \frac{x g^{\prime}(x)}{g(x)}
$$

exactly as in (14) above. Note that this is independent of the $a_{i}$ 's. 
In soft selection,

$$
s_{i}=\frac{a_{i}}{\sigma_{z}} k
$$

where $\sigma_{Z}^{2}$ is the variance of $Z$. The variance $\sigma_{i}^{2}$ of $X_{i}$ is (following the method in Section 3.1) found to be

$$
\frac{r_{i}}{s_{i}}\left(1-p_{0}\right)
$$

for all $i$. Also,

$$
\sigma_{Z}^{2}=\sum_{i} a_{i}^{2} \sigma_{i}^{2}
$$

These expressions imply

$$
\sum_{i} s_{i} r_{i}=\frac{K^{2}}{1-p_{0}},
$$

which should be compared to part 2 of Theorem 1 for haploids. Again, there is a tradeoff in that rapid substitution implies a small selection coefficient, but there is no trouble obtaining $\sum_{i} r_{i}=2$ in this model.

\subsection{Nei and Competition}

Many authors have equated hard selection with fitness being determined independent of intrapopulation competition. But Nei (1971) has derived the usual multiplicative fitnesses hard selection model while including 'competition'.

In order to sort out the role of 'competition' in hard or rank selection, it is necessary to return to the definitions. Hard selection allows both $w(0)$ and $B$ to decrease with crowding effects, and so can be made to include a population regulating mechanism. Crowding is certainly one form of competition, and hard selection does not preclude it.

On the other hand, hard selection implies that, although $w(n)$ may depend on the quantity of the competition, it does not depend on the quality of the competition. In order to illustrate this, consider the following thought experiment.

Suppose that a fruit fly population consists entirely of two types of individual, $\mathrm{A}$ and $\mathrm{B}$, where the number of inferior alleles of a type $i$ individual is $n_{i}$ and $n_{\mathrm{A}}<n_{\mathrm{B}}$. Put 1000 larvae in an environment which can support only about 100 adults. Vary the proportion of type A larvae in different vials. When the flies reach maturity, remove the (approximately) 100 survivors and compute $w\left(n_{A}\right) / w\left(n_{B}\right)$ for each vial. Hard selection demands that this quantity remains constant, independent of the quality (fraction of type B flies) of the competition.

Thus, whether or not hard selection is compatible with 'competition' depends on exactly what we mean by 'competition'. 


\section{Does nature grade on a curve?}

One of the best analogies for understanding the difference between hard and rank selection is the difference between being graded on a fixed scale and being graded on a curve. On a fixed scale, a student's grade is determined independently of the rest of the class. But when being graded on a curve, no score (except for perfection) is assured of receiving a passing mark, owing to possible superior performances by enough of the rest of the class. The question is, which scale, if either, does nature employ?

The case against hard selection is very strong. For example, although we could not know $n_{\mathrm{A}}$ or $n_{\mathrm{B}}$ in the thought experiment in Section 8 above, we certainly could find two strains of fly, $\mathrm{A}$ and $\mathrm{B}$, with one known to be more fit than the other, and perform the experiment.

It would be a great surprise if the ratio turned out to be even approximately constant. There is abundant evidence (at the phenotypic level) that survival can depend on position in the 'peck order', so that anything approximating hard selection is extremely implausible.

Yet, under rank selection, the entire genome interacts to determine an individual's rank, and therefore its fitness. Surely this is unrealistic: some components of fitness must be independent of others. This criticism of threshold rank selection was first made by Crow (1970: p. 168). In particular, if different parts of the genome are expressed at different stages of development, it is impossible for genes which are expressed late in the life cycle to influence viability at an early stage

It is fairly easy to modify the rank selection model developed here in order to incorporate rank selection in $n$ independent fitness components or at $n$ sequential stages of development. The details will be published elsewhere, but the important point is that the substitution rate $r$ can be made as large as we wish by decreasing the selection coefficient $s$ as in the rank selection models above. For a given $s$, the substitution rate $r$ turns out to be be inversely proportional to $n$ for multicomponent linear rank selection.

A few precautionary comments about experimental input need to be made. In order to test the rank selection model correctly, it is critical to understand that individuals are being ranked not according to some phenotypic character, such as size, but according to the number of inferior alleles they carry. And it is important to note that these inferior alleles are not recent mutants waiting to be purged from the population, but alleles which were previously fixed and are undergoing replacement by an adaptive evolutionary process. If any experimentation is possible in the laboratory, it will say much more about the mutation load than the process of substitution. Yet even indirect information about the process of substitution (such as the thought experiment suggested above) is undoubtedly worth gathering.

In conclusion, hard selection as the dominant mode of natural selection in adaptive evolution is completely untenable and must not be used to draw conclusions about the evolutionary process. On the other hand, although simple rank selection has unrealistic features and should not be used as the final 
resolution of Haldane's dilemma, some of its more realistic modifications, such as $n$-component linear rank selection, also allow arbitrarily rapid evolution given a small enough selection coefficient. Therefore, there is no theoretical upper bound to the rate of gene substitution, at least not for the reasons put forth by Haldane, and the neutral theory gains no support from load arguments.

\section{Acknowledgements}

I would like to thank W. Ewens, J. Murray, and J. Keener for their advice and helpful suggestions at various stages of this work. This work was supported by NSF grant DMS-8801446 as well as by the Science and Engineering Research Council of England through a grant to the Centre for Mathematical Biology, Oxford.

\section{REFERENCES}

Brues, A. M. 1964 The cost of evolution vs. the cost of not evolving. Evolution 18, $379-83$.

Brues, A. M. 1969 Genetic load and its varieties. Science 164, 1130-36.

Crow, J. F. 1970 Genetic loads and the cost of natural selection. In: Mathematical Topics in Population Genetics (K. Kojima, ed.) Berlin: Springer, pp. 128-77.

Ewens, W. J. 1972 The substitutional load in a finite population. Am. Nat. 106, 273-82.

Ewens, W. J. 1979 Mathematical Population Genetics. Berlin: Springer.

FeLLER, W. 1967 On fitness and the cost of natural selection. Genet. Res. 9, 1-15.

Felsenstein, J. 1971 On the biological significance of the cost of a gene substitution. Am. Nat. 105, 1-12.

Grant, V., \& Flake, R. H. 1974 Solutions to the cost-of-selection dilemma. Proc. Natl. Acad. Sci. USA 71 $3863-65$.

Haldane, J. B. S. 1957 The cost of natural selection. J. Genet. 55, 511-24.

Hoyle, F., \& Wickramasinohe, C. 1984 From Grains to Bacteria. Cardiff, UK: University College Cardiff Press, pp. 194-5.

Hoyle, F., \& Wickramasinghe, C. 1986 Archaeopteryx, the Primordial Bird. Sketty, Swansea, Wales: Christopher Davis, pp. 21-2.

Kimura, M. 1968 Evolutionary rate at the molecular level. Nature 217, 624-6.

Kimura, M. 1971 Theoretical foundation genetics at the molecular level. Theor. Popul. Biol. 2, 174-208.

Kimura, M. 1983 The Neutral Theory of Molecular Evolution. Cambridge University Press.

Kimura, M., \& Crow, J. F. 1969 Natural selection and gene substitution. Genet. Res. 13, $127-41$.

Kimura, M., \& Crow, J. F. 1978 Effect of overall phenotypic selection on genetic change at individual loci. Proc. Natl. Acad. Sci. USA 75, 6168-71.

Kimura, M., \& Maruyama, T. 1966. The mutational load with epistatic gene interactions in fitness. Genetics 54, 1337-51.

Kimura, M., \& Maruyama, T. 1969 The substitutional load in a finite population. Heredity 24, 101-14.

KondRashov, A. S. 1984 Rate of evolution in a changing environment. J. Theor. Biol. $107,249-60$.

Maynard Smrth, J. 1968 'Haldane's dilemma' and the rate of evolution. Nature 219, 1114-16.

Maynard SMrth, J. 1978 Evolution of Sex. Cambridge University Press. 
Moran, P. A. P. 1970 'Haldane's dilemma' and the rate of evolution. Ann. Hum. Genet. 33, 245-9.

NeI, M. 1971 Fertility excess necessary for gene substitution in regulated populations. Genetics 68, 169-84.

O'Donald, P. 1969 'Haldane's dilemma' and the rate of natural selection. Nature 221, 815-16.

Phelps, F. M. 1989 The substitutional genetic load and the neutral theory of molecular evolution. Ph.D. thesis, University of Utah.

Sved, J. A. 1968 Possible rates of gene substitution in evolution. Am. Nat. 102, 283-93.

Van Valen, L. 1963 Haldane's dilemma, evolutionary rates and heterosis. Am. Nat. 97, $185-90$.

WALLACE, B. 1975 Hard and soft selection revisited. Evolution 29, 465-73.

WILLS, C. 1978 Rank order selection is capable of maintaining all genetic polymorphisms. Genetics 89, 403-17. 